\title{
AFINA-te \\ A Healthy Lifestyle Information Website, Online Food Diary and Exercise Log Directly Towards Children
}

\author{
Nuno Guimarães ${ }^{1}$, Vera Ferro-Lebres ${ }^{2}$ and José Carlos Ribeiro ${ }^{1}$ \\ ${ }^{1}$ Faculty of Sports, University of Porto, Porto, Portugal \\ ${ }^{2}$ School of Health, Polytechnic Institute of Bragança, Bragança, Portugal \\ \{nguimaraes,jribeiro\}@fade.up.pt,vferrolebres@ipb.pt
}

\begin{abstract}
Keywords: Children, Exercise, Nutrition, Online, Website.
Abstract: Childhood obesity is according to the World Health Organization one of the most concerning problems today. Educating children to a healthier lifestyle is a difficult task due to the lack of interest or concern that they demonstrate. The interest that children have in technology and the time they spent online in games or simply surfing the web may be seen as an opportunity to instill knowledge about healthy eating and healthy lifestyle. There are already several online health counseling websites but it seems to exist a lack of such platforms directly towards to children. Afina-te website is an online platform that aims to monitor and educate children to a healthier lifestyle through the exposition of information, interactive applications and educational games. It is also capable of provide feedback about what users eat and the exercise they practice. This paper describes the development and resulting health counseling website.
\end{abstract}

\section{INTRODUCTION}

Childhood obesity is a global problem with great concern for public health. The body mass index (BMI) is used to define overweight and obesity amongst children and teenagers. When the BMI is higher or equal to $95^{\text {th }}$ considering age and gender then it is defined as obesity. When is between $85^{\text {th }}$ to $94^{\text {th }}$ it is considered overweight (Barlow et al., 2007). The World Health Organization (WHO) pointed out that in 2012, 44 million children were overweight (WHO, 2014). In Portugal, recent studies from the Directorate-General of Health (DGS) concluded that approximately $35 \%$ of 6 to 8 year old children were overweight (DGS, 2013). Obesity can also lead to several health problems in short and long term periods (Daniels, 2009). To prevent such problems, children must be aware about the consequences of poor eating habits and lack of exercise practice. The growing interest of children in new technologies and the learning they take from it (Akhter, 2011) could be useful to instill healthy lifestyle knowledge. The use of technologies as a way to spread healthy lifestyle habits is not new. The study by Jarvinen (Järvinen, 2007) shows already several websites with free or paid subscriptions that allow users to receive online counseling about eating habits and physical exercise. More recently these In- ternet services have been using mobile applications to facilitate the insertion of nutrition and exercise data of users (e.g. My Fitness Pal (MyFitnessPal, 2014) and My Net Diary (MyNetDiary, 2014)). The use of smartphones with these services allows an easier and quicker way to submit information and retrieve feedback on the eating and exercise behaviors of users. However, the target users for this type of websites are adults since it is of their own interest and initiative to stay healthy. Adults are willing to make the effort to fill food diaries, report exercise habits and other type of information as long as they have the feedback about their nutrition and physical condition. In order to get the same goal for children, the current websites will not work adequately since their display of information and user interface design is not suitable for the age group (Taslim et al., 2009). The way children report their nutrition habits must be simple, intuitive and enjoyable in order to make them repeat several times (by their own initiative) that process. The way that information is displayed must also be taken in concern since children tend to prefer larger letter fonts and images, animations and audio to substitute text information (Bruckman and Bandlow, 2003). There is already some work attempting to promote this healthy lifestyle education and simplify the process of entering food and sport practice infor- 
mation. Regarding healthy lifestyle habits there are several works trying to spread knowledge in this area through educational games. Grocery Hunter is an educational mobile game that intends to teach healthy eating habits to children and fight obesity by making them do healthy food choices when they are at the grocery store (Kim et al., 2011). The application consists in a search game where the users receive tips from a cartoon regarding a specific food item. When the player finds it, he points out the mobile camera to it and the application will provide feedback whether it is the correct choice or the wrong one. Another work with the same purpose is the MunchCrunch. This quiz team game with healthy eating questions targets the teenagers and intends, in a fun way, to teach them more about good nutrition habits (Mansour et al., 2009) . It becomes clear the contribution that educational games have in children and how can enhance their learning (Alemi, 2010; Virvou et al., 2005). It is also important for children applying that knowledge to their eating and exercise habits and see how they are damaging or benefiting their health. So it is important to have an online food and exercise log so they can monitor both of those health components. The GetFit website was developed with the purpose of monitoring activity and eating habits for patients of the Children Fitness Clinic (CFC) at the University of Virginia. The counselors can monitor patient's activity and nutrition habits between face-to-face sessions through online diaries that the patients fill. The website also contains information, links and educational games regarding healthy habits (Olson et al., 2010). Due to its development purpose, Get Fit website was not design to provide feedback to their users on their daily habits. It is comprehensive since it intends to be used between patient-counselor meetings and it is here where the patient receives the necessary feedback about their habits. Another problem is that patients must fill their diary and activity logs. We must question if they would still go to the website and fill the logs based only in their interests and motivation. The goal of this paper is to describe the basis and the process of building the Afina-te platform. Afina-te is the portuguese acronym for "physical activity and nutrition information for adolescents". The website is a health counseling Internet service specifically directed to adolescents and its main goals are to promote a healthy lifestyle and provide counseling and feedback depending on each user physical characteristics (like weight, gender and height), eating journals and physical activities. It is also the goal of Afinate to make an intuitive and suitable interface, keeping children interested in providing their daily nutrition and activity habits.

\section{RESEARCH APPROACH}

The main goal of Afina-te website was to provide a specific and feasible alternative for kids of the current nutrition and fitness online platforms. The project group consists in nutritionists, dietitians, exercise specialists and a web programmer. The project started with the development of the Afina-te database. MySQL system was used to create and manage users information like credentials, personal information (i.e. address, cellphone, email), physical measurements (i.e. weight, height), school and activities schedule, food logs and exposition time. This last one was one of the requirements since the beginning of the project so we could try to understand where users spend more and less time (consequently improving the pages they rarely visited). Exposition time is controlled in the following way: every time a user accesses a page in the Afina-te website, a timer starts to run in the server. When he leaves the page, the time he spent there, the user id and the name of the page is stored in the database. The database was designed to include information relatively to food items and quantities. Afina-te food database includes over 800 items and 2000 quantitative measures regarding those items. Most of the items are retrieved from the Portuguese Food Composition Table (FCT, 2014) for precision purposes. However there is a small percentage that was retrieved directly from food labels. The design phase began with the construction of a basic website structure and suffered major changes along the entire project, not only due to the feedback we were taking from test users, but also from the study of web design trends. The information presented in the several sections has also change during the development process since writing adaptations and text to images replacements were frequently made to create an interesting website for the users age range. Nutrition and exercise specialists wrote all texts from the site in Portuguese. The type of writing was also adapted to the age group in question. It was also defined at the beginning of the project the importance of establishing a limit time of 60 minutes per day, so the website itself does not encourage users to exceed the recommendations of screen time (Kaneshiro et al., 2014).

\section{RESULTS}

\subsection{Site Design}

The site design consists in a navigation menu that is always fixed at the top of the page as figure 1 shows. This menu has several buttons represented by icons. 
The icons illustrate the section of the website the button leads to. However, since the icon may not provide enough information on the section topic, a description will come up on hovering the button (in the figure the descriptions is "Take a Change"). There are two more elements on the right side of the menu. The first one is a counter of how many users are online at the same time (in this case 1) and the other is the remaining time the user has for the day. Below the navigation

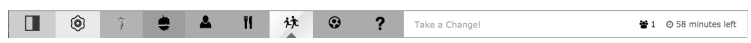

Figure 1: Navigation Menu.

menu we have the page content that varies according to the section where the user is. Across all Afina-te website we use a flat design. This particular kind of design was made popular lately due to its use in operating systems (such as Windows 8 and OSX) and several smartphone applications. The main features of flat design is the absence of shadows, gradients and textures that are used for giving a three dimensional effect (TheNextWeb, 2014).

\subsection{Site Features}

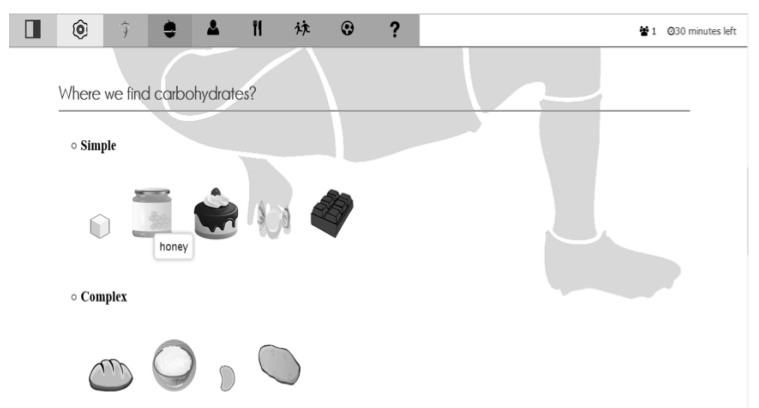

Figure 2: Example of content page.

The website is divided in 3 main sections: My Personal Space, Nutrition and Exercise. Nutrition section has content pages about energy, nutrients, food wheel, food and fitness, healthy body and healthy recipes (figure 2 shows an example). All this pages were designed to display the information using the maximum of interactivity, images and animations possible. As an example, in each nutrient page we have specified the necessary daily intake for each user based on the data stored. We also have images to illustrate where the nutrients are found, animated food wheels and interactive recipe books. Below we describe the most interactive pages in the nutrition section.

- Example Label - an interactive label where the user can click in the different sections and learn about them through information displayed in popup windows;

- Label Inspector - in this section the user is asked about concrete information on the nutritional values and ingredients presented on the label. He must click in the label section where the answer is. As an example the user could be asked which ingredient is present in the largest amount. The user must know (from the information provided in the example label) that the ingredients are ordered by the amount included in the product;

- "Who Wants to Be Healthy?" - is a similar game to the popular TV quiz show "Who wants to be a millionaire". The user must answer correctly to 15 questions of multiple choices using the knowledge he acquired from other sections of the site. The user has 3 help options: 50-50, call to a friend and public help. The first one random removes 2 wrong options of the total 4 . The other two are probabilistic choices. The more closer the user is to the victory the less probable is to get the correct answer from the "help".

The exercise section contains information about the different exercise components such as strength, flexibility and aerobic endurance. It also includes a section with information about sports such as football, basketball and tennis. It is our goal to update this section with new sports when Afina-te is fully operational (in order to keep users interested in the site). In the exercise section the user can also ask questions about this topic. These questions are available to all users so it should not be personal questions (there is a section for those kinds of questions that we will discuss later). Exercise specialists who collaborate in the site will give the answers to the questions asked. The third section of Afina-te website is the "My personal space" section. It is in this section that the user provides all the information about his daily meals and weekly activities. It is also in this section that the user retrieves the most part of the feedback. We will describe the different pages of this section in more detail.

\subsubsection{My Personal File}

In this page the user can check his personal data that was given in the register (like address, birth date and cellphone). It can also see his physical data such as weight, height and fat percentage. This information is displayed in text form. The rest of the page consists in the following graphs: user fat percentage in comparison with the recommended values, daily intake in comparison with recommended intake, daily energy expended in comparison with energy intake, monthly energy expended in comparison with energy 
intake, daily nutritional (fat, protein, fiber and lipids) consumption in comparison with daily recommendations. The previous daily and monthly nutritional information graphs can be consulted through navigation buttons that allow selecting a specific date. The daily information is only provided if such entries exist in the food $\log$.

\subsubsection{Online Food Diary}

To add a meal to the food log, the user must first fill information relatively to date, hour, type (i.e. breakfast, lunch,...), local and company. After the submission, a new meal will be added to the meal list. The user can them add food to that meal, delete the meal, see the list of food items already added and see the nutritional graph of the meal. The "add food item option" has 2 different types of search: search by name (where options are shown as the user starts to type the name of the food) and search by food group. There is also an option to copy a previous meal (since some meals, like breakfast, are very similar) and a most frequently used food item selection (where the most 5 used items are shown for selection). In all options the user must select the cooking method of the food (grilled, roast, cooked...), a measure ( 1 spoon, $1 / 2$ plate...) and quantity ingested. The user can add meals up to 3 days before the current day. He can also generate a daily report in PDF with the correspondent nutrition information for printing purposes.

\subsubsection{My Weekly Schedule}

In this section the user can define his weekly schedule. This includes classes and extra curricular activities. The user can add or remove activities. Each time a user adds a non-sport activity he must fill a form with the name of the activity, start and end hour and day of the week. He may also add a description. In case user selects a sport activity, Afina-te website has a database with more than 200 sports and respective METS. METS is the unit of energy cost and it allows the calculation of energy expenditure for a particular activity (Mackenzie, 2003). This way, the graphs presented in "My Personal File" section are more precise since they retrieve data from the user daily activities to calculate the daily energy recommendation.

\subsubsection{My Goals}

Each user can define up to 3 different goals to improve his health. The goals can be "loose weight", "increase muscular mass" and "increase cardiorespiratory endurance". The user must also establish a limit number of days to fulfill each goal. When it is submitted, the nutritionists and exercise specialists who collaborate in the Afina-te website must validate or reject the goal (if they think that is not achievable). They can also leave advices to help the users fulfill them.

\subsubsection{My Photos}

This page is the user gallery to upload his meals, exercises and other nutritional and sports related photos. This section is mainly to give the user some social network features although in this first stage, the photos can't be shared with others. It also allows storing photos of the interactive food log whose functionalities we will mention below.

\subsubsection{My Questions}

This is where each user can ask the nutritionists and exercise specialists working in Afina-te about their doubts and concerns. The questions and answers will be private so other users can't access them.

\subsubsection{My Parents Questions}

The parents will have a special access so they can keep track of the information that their children are exposed to. However, such information will be relatively only to the contents of the pages and no personal information of their children will be shown. Thus, we guarantee the confidentiality of users and that they are not constrained on the information they share. This is the only section exclusively to the parents and it is similar to "My Questions". This is where they can ask about any aspect of the website or receive help tips to improve their children health habits.

\subsubsection{Interactive Food Diary (What Did I Eat)}

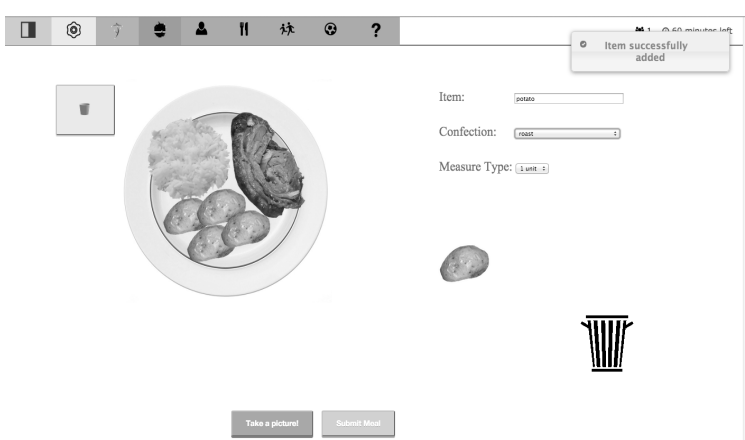

Figure 3: Example of the interactive food log.

In this section we provide a fun and interactive way to fill the online food journal. The user can search the food in the item box that contains an 
auto complete functionality (it will make suggestions based in the inputted letters). Once the item is found the user must select the cooking method and the measure type. In the end, an image of the correspondent item will show up. The user must drag the item and place it in any part of the plate. The same item can be added multiple times since what it is dragged to the plate is a clone of the original. To delete an item from the plate the user should drag it to the trash bin icon. To add drinks the user must click in the glass button to open a popup window. Then he must select the type of drink and measure using the "+" and "-_" to fill and empty the cup (the drink in the cup refers to the quantity that the user ingested relative to the measure he selected). In the end he clicks "OK" and the drink is added to the meal. It is possible to submit multiple drinks by repeating the process. The user can also add soup to the meal by clicking in the respective button. A similar popup window will appear where he can select the type of soup and measure. Multiple soups can be added to the meal. With this interactive application, the user can construct a very similar plate to the one he had. When the plate is complete the user clicks the submit button and must complete the meal information such as date and hour. The user can also take a picture of the plate that it will be stored in the his personal gallery.

\subsubsection{What I Should Eat}

This page is very similar to the "What Did I Eat" page, however it allows users to freely construct a meal in the same way than before and get feedback (through text and graph) on the adjustment of that meal in their daily intake. This means that the user can learn if his daily meals are adjusted to his daily nutritional intake or not.

\section{DISCUSSION}

Afina-te website is a new approach to an old issue: the fight against obesity. There are already several online websites to help users to improve their health. However very few are directly targeted to children and the ones that are, have an old design and lack of feedback due to the context that they were built (Olson et al., 2010). Afina-te intends to explore the relation of children with technology to instill some healthy lifestyle knowledge, making them use part of their daily screen time to learn and receive feedback about their nutrition and exercise habits. We predict that the way information is presented and the several interactive pages of the website will raise interest enough so users continue visiting it and filling the food and exercise logs. It is also important to update the platform with new content on a regular basis to keep users motivated to revisit it. The website developed takes in consideration the several design studies made. Nevertheless, children were not involved in the design process being only used for occasional feedback on a particular section or page. This may present a limitation to the correct and intuitive use of the website since children should be involved since the beginning of the design and content development. Also, since the age of the users differs widely, the content and design may not be ideal for every user. However, a pilot study is scheduled in one portuguese school in different age users so we can have some feedback relatively to design and content. We intend to determine which parts are suitable for all users and which parts are needed to adapt for different ages and gender. Based on the feedback we will create different designs for the website which will be displayed according to the user's age. We tried to make a more general design for the pilot study and we will adapt it accordingly to the feedback received. In America, the use of smartphones in children has increased significantly (Rideout, 2013) so the development of a smartphone application to complement the website would be useful to simplify the input of information (since it would be done on the fly) and consequently provide almost instantaneous feedback about the logs submitted. The absence of such application could be seen as a limitation to achieve more precision in the logs since children tend to easily forget some food if they do not write it down immediately after the intake.

\section{CONCLUSIONS}

Web-based interventions seem to allow users to access information at their own pace (time, place, cognitive assimilation of information etc.) (Griffiths et al., 2006); permitting a better exposure and a more intensive cognitive processing (Brug et al., 2003). In particular, for adolescents also other advantages have been identified, the effect of novelty; the attractiveness of the internet to this age range; and the use of online communication as one of the main forms of communication for young people (Griffiths et al., 2006). Moreover the use of technologies in interventions regarding health knowledge has been recommended (USD, 2010), considering that personal feedback on health practices together with tailored information about the best health choices, has considerable potential to communicate a personal need to change (USD, 2010). In this paper we present a health coun- 
seling web platform that, unlike other similar websites, the target population are children. Each user has access to easy to read information about nutrition and exercise presented in simple text, images, animations, interactive applications and games. They also have access to feedback about their health through the filling of interactive online food logs, exercise schedules and chatting with nutrition and exercise specialists. Afina-te is a learning platform for children consequently leading them to better understand, apply and monitor healthy behaviors, therefore preventing and help treating obesity and overweight. In the future, we intend to make an intervention in several public schools to determine if the site fulfills its purpose. It would be also interesting, in a future step, the creation of a smartphone application for the Afina-te platform making it a full health counseling bundle (website + smartphone application) for children.

\section{ACKNOWLEDGEMENTS}

Project supported by: PTDC/DTP-DES/1328/2012 (FCOMP-01-0124-FEDER-028619); and Research Center supported by: PEst-OE/SAU/UI0617/2011.

\section{REFERENCES}

(2010). National action plan to improve health literacy. United States: U.S. Department of Health and Human Services.

(2013). Portugal healthy eating in numbers (Portugal alimentação saudável em números) - 2013. DireçãoGeral da Saúde. Accessed: 2014-10-07.

(2014). Food composition table (tabela da composição de alimentos). Accessed: 2014-10-1.

(2014). World health statistics 2014. World Health Organization. Accessed: 2014-10-07.

Akhter, P. (2011). An extensive review on children's learning process through their use of digital technology at home. In 5th International Conference on Software, Knowledge Information, Industrial Management and Applications.

Alemi, M. (2010). Educational games as a vehicle to teaching vocabulary. Modern Journal of Applied Lingusitics, 2(6):425-438.

Barlow, S. E., MD, MPH, and the Expert Committee (2007). Expert committee recommendations regarding the prevention, assessment, and treatment of child and adolescent overweight and obesity: summary report. Official Journal of the American Academy of Pediatrics, 120:S164-S192.

Bruckman, A. and Bandlow, A. (2003). Human-computer interaction for kids. In Jacko, J. A. and Sears, A., editors, The Human-computer Interaction Handbook, chapter Human-computer Interaction for Kids, pages 428-440. L. Erlbaum Associates Inc., Hillsdale, NJ, USA.

Brug, H., Oenema, A., and Campbell, M. (2003). Past, present, and future of computer-tailored nutrition education. The American Journal of Clinical Nutrition.

Daniels, S. R. (2009). Complications of obesity in children and adolescents. International Journal of Obesity, 33:S60-S65.

Griffiths, F., Lindenmeyer, A., Powell, J., Lowe, P., and Thorogood, M. (2006). Why are health care interventions delivered over the internet? a systematic review of the published literature. J Med Internet Res, 8(2): 10

Järvinen, P. (2007). Hybrid media in personal management of nutrition and exercise. Technical Report 656, VTT. VTT Publications.

Kaneshiro, N. K., Zieve, D., and A.D.A.M. (2014). Screen time and children.

Kim, H., Kogan, A., Dasgupta, C., Novitzky, M. M., and Do, E. Y.-L. (2011). Grocery hunter: a fun mobile game for children to combat obesity. In Gross, M. D., Nunes, N. J., Do, E. Y.-L., Brewster, S. A., and Oakley, I., editors, Tangible and Embedded Interaction, pages 317-320. ACM.

Mackenzie, B. (2003). Mets. Accessed: 2014-10-1.

Mansour, A., Barve, M., Bhat, S., and Yi-LuenDo, E. (2009). Munchcrunch: a game to learn healthy-eating heuristics. In Paolini, P. and Garzotto, F., editors, IDC, pages 166-169. ACM.

MyFitnessPal (2014). My fitness pal. Accessed: 2014-0923.

MyNetDiary (2014). My net diary. Accessed: 2014-09-23.

Olson, A., Bond, M., Aspinall, B., Williams, B., Stoecker, E., Jianping, W., Hellems, M., and Guerlain, S. (2010). Supporting food and exercise monitoring for overweight and obese children. In Systems and Information Engineering Design Symposium (SIEDS), 2010 IEEE, pages 233-238.

Rideout, V. (2013). Zero to eight - children's media use in america 2013.

Taslim, J., Adnan, W. A. W., and Bakar, N. A. A. (2009). Investigating children preferences of a user interface design. In 13th International Conference, HCI International 2009.

TheNextWeb (2014). The history of flat design: How efficiency and minimalism turned the digital world flat.

Virvou, M., Katsionis, G., and Manos, K. (2005). Combining software games with education: Evaluation of its educational effectiveness. Educational Technology \& Society, 8:54-65. 